\title{
Adaptive bonding technique for precise assembly of three-dimensional microstructures
}

\author{
Sang-Hu Park \\ School of Mechanical Engineering, Pusan National University, Busan 609-735, Korea \\ Jun-Ho Jeong, ${ }^{\text {a) }}$ Dae-Geun Choi, Ki-Don Kim, Ali Ozhan Altun, and Eung-Sug Lee \\ Nano-mechanical System Research Center, Korea Institute of Machinery and Materials, Daejeon 305-343, \\ Korea \\ Dong-Yol Yang \\ Department of Mechanical Engineering, Korea Advanced Institute of Science and Technology, \\ Daejeon 305-701, Korea \\ Kwang-Sup Lee \\ Department of Polymer Science and Engineering, Hannam University, Daejeon 306-791, Korea
}

(Received 9 January 2007; accepted 13 May 2007; published online 6 June 2007)

\begin{abstract}
Precise fabrication of three-dimensional (3D) self-standing microstructures on thin glass plates via two-photon induced polymerization (TPP) has been an important issue for innovative 3D nanodevices and microdevices. However, there are still issues remaining to be solved, such as building 3D microstructures on opaque materials via TPP and being able to implant them as functional parts onto practical systems. To settle these issues simply and effectively, the authors propose a contact print lithography (CPL) method using an ultraviolet-curable polymer layer. They report some of the possibilities and potential of CPL by presenting their results for transplanting 3D microstructures onto large-area substrates and also their examination of some of the effects of the process parameters on CPL. (C) 2007 American Institute of Physics. [DOI: 10.1063/1.2746085]
\end{abstract}

Two-photon induced polymerization (TPP) has been intensively studied for the past ten years for a wide range of three-dimensional (3D) applications, including nanofabrication and microfabrication. ${ }^{1-7}$ Such diverse materials as metals, ceramics, and high strength polymers are used in fabrications for various high-value-added applications via TPP., Further, research on the development of various schemes for effective 3D fabrication are continuously conducted as TPP is expected to be applied to more and more widely ranging processes and applications. ${ }^{10-13}$

Despite this promising potential of TPP, there are still important issues to be settled, including how 3D microstructures can be fabricated on opaque substrates, such as silicon wafers and metal layers, regardless of the surface flatness, and how 3D structures can be placed on microsystems to realize their specific function in the system. Although direct fabrication of 3D microstructures on opaque materials and inside systems containing macroscale components should be possible using TPP, precisely controlling a tightly focused beam on a large area with a nanostage is generally cost prohibitive. Further, most research has required immersion oil (refractive index of 1.51) in front of the objective lens to increase the numerical aperture and generate the smallest possible spot size. A thin transparent glass plate is then necessary to isolate the immersion oil from the photocurable resin, explaining why most 3D microstructures have been built on thin glass plates rather than on opaque materials [see Fig. 1(a)]. Thus, another approach for manipulating 3D microstructures is required. We therefore propose a contact print lithography (CPL) technique to transplant 3D micro-

\footnotetext{
a) Author to whom correspondence should be addressed; electronic mail:
} jhjeong@kimm.re.kr structures fabricated by TPP onto other materials, regardless of their transparency. With CPL, relocating, implanting, and immersing 3D microstructures onto any substrate is possible, suggesting the construction of a practical microsystem built of functional 3D components. We also present the CPL pro-

(a) Fabrication of a 3D microstructure

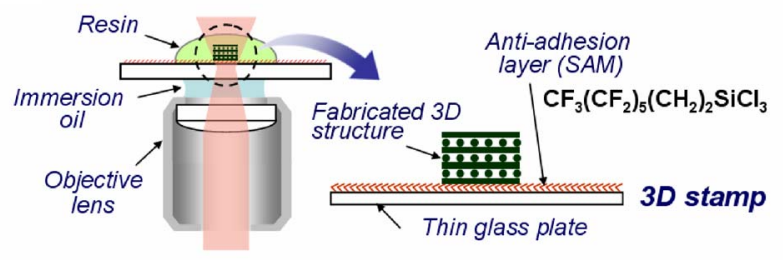

(b) Positioning \& Joining

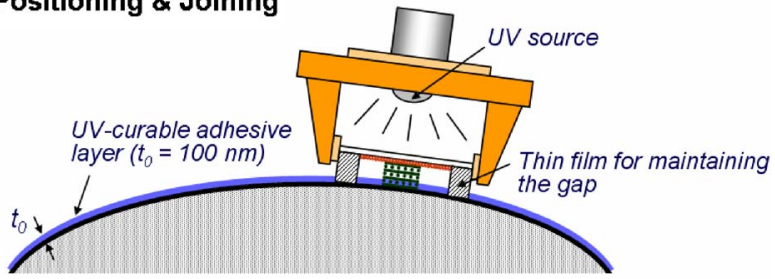

(c) UV-exposure and Release the 3D stamp

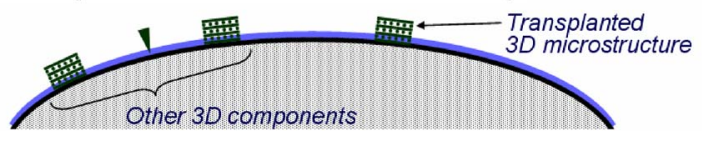

FIG. 1. (Color online) Schematic illustration of the three steps of CPL. (a) Fabrication process of a 3D stamp using TPP. Before 3D fabrication, a SAM (FOTS) was deposited on the glass plate to reduce the adhesiveness between the glass plate and the 3D microstructures. (b) Positioning of the stamp on the adhesive layer to join the 3D microstructures to the substrate by UV exposure. (c) Careful removal of the stamp using tweezers. 

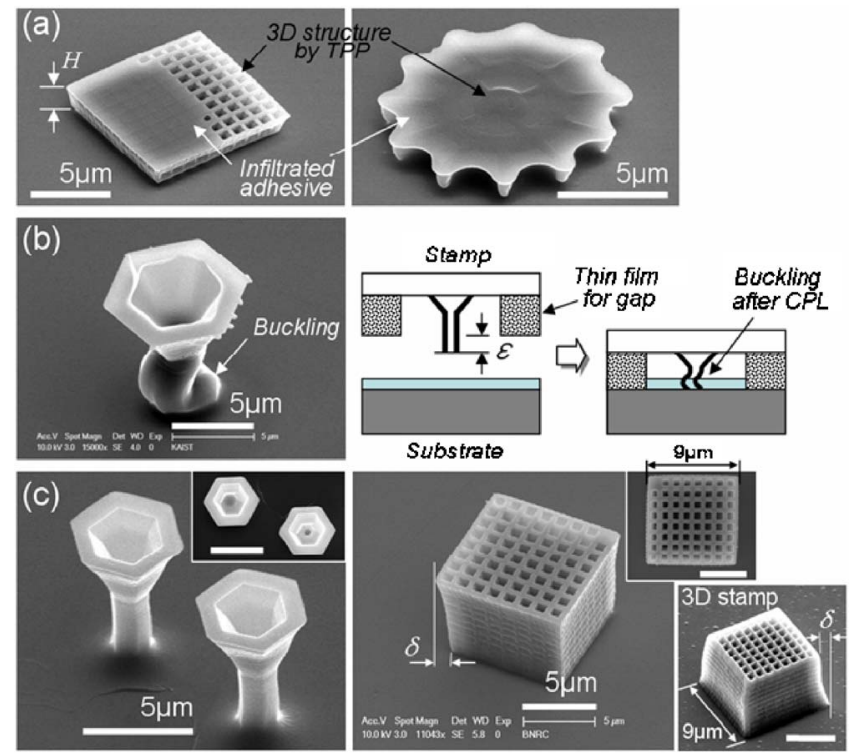

FIG. 2. (Color online) Scanning electron microscopy (SEM) images of transplanted 3D microstructures on a substrate. (a) If the height $(H)$ of the 3D structure was too small, the adhesive resin infiltrated the 3D structure by capillary forces. (b) When the height of a 3D microstructure was larger than the gap (film thickness), the structure buckled during CPL. (c) Well-standing pillars with hexagon heads and a woodpile structure by controlling the gap. The shrinkage volume $(\delta)$ shown at the top of the stamp was reverse transplanted onto the substrate. Their dimensions were not changed after CPL. The upper-right images are top views of the structures, and all scale bars are $5 \mu \mathrm{m}$.

cess parameters for the precision transplantation of 3D microstructures onto a substrate.

There are three fundamental steps when using CPL to transplant 3D microstructures onto a macroscale substrate, as schematically depicted in Figs. 1(a)-1(c). First, a 3D stamp is prepared for contact printing. A self-assembly monolayer (SAM) is then vapor deposited onto the glass plate using an ion method to reduce the adhesive force between it and the $3 \mathrm{D}$ microstructure. The reverse shape of the 3D structure is fabricated using TPP on the plate that is utilized as a carrier plate. Then it is positioned on a substrate coated with a thin, ultraviolet (UV)-curable, adhesive layer [see Fig. 1(b)]. To effect the contact between the 3D structure and the adhesive layer, the circumference is pulled down with a 1 bar vacuum. Next, the bonding layer is exposed to UV light to be cured and to bond the 3D structure on the substrate and the plate is then carefully removed. We have set up an optical laser system for preparing the 3D stamps; the used materials, detailed specifications, and operation of the system were explained in previous work of some of the authors. ${ }^{10-13}$ To evaluate the usefulness of CPL, we transferred some 3D microstructures onto the surface of a Si wafer. The adhesive layer on the substrate was a commercial UV-curable resin, AMO NILMMS10 (from AMO GmbH). It was spin coated with a thickness of approximately $100 \mathrm{~nm}$ onto the surface of the $\mathrm{Si}$ wafer before the CPL. To obtain good conformal contact with the $3 \mathrm{D}$ microstructures, the adhesive layer must be of uniform thickness, but the actual thickness is not critical within the range of $1-200 \mathrm{~nm}$. If the height of the 3D structure is very low, capillary force can easily drive the adhesive into the 3D structure during CPL [see Fig. 2(a)]. The maximum infiltrated height $\left(h_{\max }\right)$ is dependent on a narrow gap

$(L)$ in the structure, the surface tension $(\gamma)$ at the adhesive/air Downloaded 13 Apr 2011 to 143.248 .233 .201 . Redistribution subject to AIP interface, and the contact angle $(\theta)$ at the adhesive/ microstructure interface, which gives $h_{\max }=(2 \gamma \cos \theta) / \rho G L$; here, $\rho$ is the density of the adhesive and $G$ is the gravitational constant. ${ }^{14}$ Therefore, the height of 3D microstructures should be higher than the value of $h_{\max }$ for the prevention of full infiltration into the structure, with the height of $2 \mu \mathrm{m}$ of a woodpile having the gap of $700 \mathrm{~nm}$, that was infiltrated to its top surface [see left of Fig. 2(a)]. For the reduction of $h_{\text {max }}$, the low surface tension and high density of an adhesive are required.

To maintain the crucial control of the gap between the stamp and adhesive layer, we matched the film thickness with the height of the 3D microstructures. Differences between the film thickness and 3D microstructure heights made stable transplantation difficult: if the film thickness was larger, transplantation was impossible due to no contact between the structure and the adhesive. Conversely, if the height of the 3D structure was much larger, the structure buckled during transplantation from the conformal contact pressure due to the amount of the difference $(\varepsilon)$ [see Fig. 2(b)]. In Fig. 2(c), two pillars with hexagonal heads and a woodpile structure are self-standing on the substrate after the CPL. Transplanting to reposition of the structures was performed using the same procedure and a controlled film thickness. The top surfaces appear completely flat since they were attached to the flat surface of a glass plate (carrier plate) before the CPL. Also, as shown in the rightmost image of Fig. 2(c), the woodpile structure on the stamp had a shrinkage volume $(\delta)$ on its top that was reverse transplanted onto the surface of the substrate. These show the 3D microstructure transplantation from the carrier plate to the substrate. Dimension changes of the 3D microstructures before and after CPL were not observed. As shown in Fig. 2(c), the edge size of the top surface in the transplanted woodpile is also $9 \mu \mathrm{m}$, compared to the edge size of the bottom surface of the structure before CPL. The height of the structure after CPL is reduced by as much as the amount of immersion into the adhesive layer (in this case, about $100 \mathrm{~nm}$ ). This indicates that there is no elastic recovery of the structure after transplantation. By this reason, precise transplantation of 3D microstructures into a system is possible.

One of the most important CPL contributors to stable transplantation is the prefabrication SAM antiadhesion treatment on the glass plate. In this work, $(1 \mathrm{H}, 1 \mathrm{H}, 2 \mathrm{H}, 2 \mathrm{H}-$ perfluoro-octyl)trichlorosilane (FOTS), which has a molecular formula of $\mathrm{CF}_{3}\left(\mathrm{CF}_{2}\right)_{5}\left(\mathrm{CH}_{2}\right)_{2} \mathrm{SiCl}_{3}$, was used as SAM for the reduction of adhesion force. ${ }^{15}$ We vapor deposited the SAM layer using our own laboratory-built equipment (AVC100). A full SAM deposition takes about $15 \mathrm{~min}$ at 4.5 torr with the precursor and argon at room temperature, where the maximum contact angle between a droplet of water and the SAM treated surface was near $105^{\circ}$, which was not increased with much more deposition of FOTS, and the contact angle $\left(\theta_{c}\right)$ was varied nonlinearly, depending on the deposition pressure and time: $\theta_{c}=65^{\circ}$ for 0.1 torr, $\theta_{c}=90^{\circ}$ for 0.5 torr, $\theta_{c}=100^{\circ}$ for 2.0 torr, and $\theta_{c}=105^{\circ}$ for 4.5 torr under the same deposition time $(15 \mathrm{~min})$. If the SAM was not deposited on the glass plate, the adhesion force between the TPPfabricated 3D structure and the glass plate was too strong, leading to a release failure from the glass plate. To qualitatively evaluate the effect of the antiadhesion layer, we conducted a simple test, depicted in the insert of Fig. 3(a), where license or copyright; see http://apl.aip.org/about/rights_and_permissions 

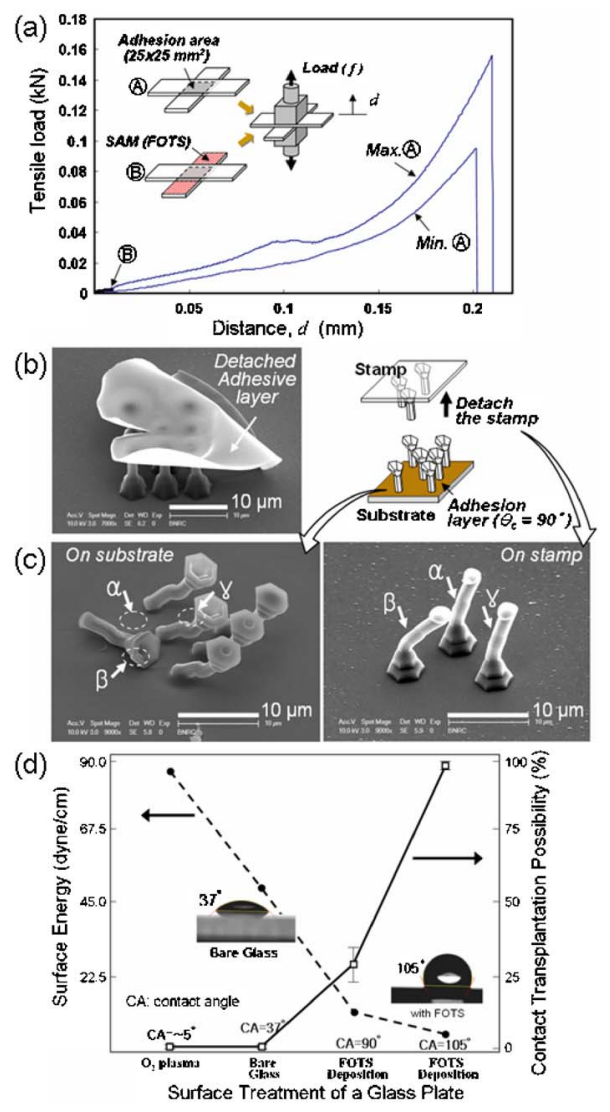

FIG. 3. (Color online) Comparison of adhesive forces with and without antiadhesion treatment. (a) Preliminary test results for evaluating the effect of antiadhesion treatment. (b) SEM images of 3D stamps after CPL without the SAM before 3D microfabrication by TPP. Part of the adhesive peeled off and attached itself to the contact surface of the stamp. (c) When the SAM was incompletely deposited onto the stamp, partial transplantation of the 3D microstructures occurred due to the detaching forces, which led to structure distortion. (d) Statistical result on the possibility of stable transplantation.

the adhesive force between glass plates with and without a SAM is shown. Tests were performed using glass plates, $75 \times 25 \times 1 \mathrm{~mm}^{3}$ in size. Some of the plates were then prepared with only an adhesive layer coating, while others were joined using the same adhesive layer after the SAM deposition on one of the pairs. The maximum loads $(f)$ at the moment of separation of the adhesion layer between the plates were measured over the range of $0.096-0.156 \mathrm{kN}$ $(0.153-0.249 \mathrm{MPa})$ for the non-SAM plates; however, when the SAM was deposited fully (in the case of $\theta_{c}=105^{\circ}$ ), the maximum loads were reduced dramatically to nearer $0.002 \mathrm{kN}(0.003 \mathrm{MPa})$ for all cases [see Fig. 3(a)].

We also investigated a practical phenomenon in CPL without an antiadhesion layer before 3D microfabrication. As shown in Fig. 3(b), the UV-cured adhesive layer was partially detached from the surface of the substrate and attached to the contact surface of the 3D microstructures on the stamp due to the strong adhesive force between the microstructure and plate. For the 3D microstructures composed of $3 \times 3$ pillars with hexagonal bottoms, the contact area with the glass plate was four times larger than that with the bonding layer, which can lead to a much higher adhesive force between the 3D structures and the glass plate. Further, if the SAM deposition is incomplete (where $\theta_{c}$ is much less than $105^{\circ}$ ), the adhesion force between the $3 \mathrm{D}$ microstructures and the glass plate may still be too strong, leading to prob- lems in transplanting the 3D structures onto the substrate. Figure 3(c) shows an abnormal result, the partially transplanted 3D micropillars and the remains on the stamp after CPL when SAM was not deposited completely on the glass plate (note that the structures were distorted during separation of the stamp from the substrate; in this case, the $\theta_{c}$ was about $90^{\circ}$ ). For statistical analysis on precisely transferred micro-objects, we have evaluated the percentage of stable transplantation using microdot arrays, which were composed of 100 dots each having a volume of $10 \mathrm{nl}$, under various surface conditions of a glass plate: $\mathrm{O}_{2}$ plasma treatment, bare glass, and FOTS deposition by 0.5 and 4.5 torr. As shown in Fig. 3(d), nearly all cases were transplanted with complete deposition of SAM [contact angle $(\mathrm{CA})=105^{\circ}$ ]. However, in the cases of without SAM (bare glass) and incomplete deposition of SAM (CA was near $90^{\circ}$ ), the possibility of transference was dramatically reduced to $0 \%-30 \%$. From this statistical analysis, it is known that the surface treatment of a glass plate may be the most important factor for the practical use of CPL.

In summary, there are numerous advantages when CPL is used to fabricate practical devices consisting of various nanoscale to macroscale components. It is possible to assemble nanodetailed 3D shapes onto an opaque substrate and to construct a large-scale system with local 3D functional microstructures. Three-dimensional patterns on a waved surface can also be readily fabricated by CPL. Limitations in 3D fabrication by TPP can be circumvented using CPL. CPL makes it feasible to fabricate innovative microsystems containing 3D microstructures, such as 3D mixers or filters inside microchannels or 3D photonic crystals inside optical devices.

This research was supported by a grant in aid for the National Core Research Center Program from MOST/ KOSEF (R15-2006-022-01001-0) and supported by a grant (06K1401-00210) from the Center for Nanoscale Mechatronics and Manufacturing supported by MOST of Korea. D.Y.Y. and K.-S.L. are grateful for Grant No. (M1050300021705M0300-21700) and the BK21 program.

${ }^{1}$ S. Maruo, O. Nakamura, and S. Kawata, Opt. Lett. 22, 132 (1997).

${ }^{2}$ S. Kawata, H. B. Sun, T. Tanaka, and K. Takada, Nature (London) 412, 697 (2001)

${ }^{3}$ H. B. Sun, K. Tanaka, and S. Kawata, Appl. Phys. Lett. 80, 3673 (2002).

${ }^{4}$ K. D. Belfield, K. J. Schafer, Y. Liu, J. Liu, X. Ren, and E. W. Stryland, J.

Phys. Org. Chem. 13, 837 (2000).

${ }^{5}$ J. M. Lourtioz, Nat. Mater. 3, 427 (2004).

${ }^{6}$ P. Galajda and P. Ormos, Appl. Phys. Lett. 78, 249 (2001).

${ }^{7}$ H. B. Sun, K. Takada, and S. Kawata, Appl. Phys. Lett. 79, 3173 (2001).

${ }^{8}$ A. T. Pham, D. P. Kim, T. W. Lim, S. H. Park, D. Y. Yang, and K. S. Lee, Adv. Funct. Mater. 16, 1235 (2006).

${ }^{9}$ A. Ishikawa, T. Tanaka, and S. Kawata, Appl. Phys. Lett. 89, 113102 (2006).

${ }^{10}$ S. H. Park, S. H. Lee, D. Y. Yang, H. J. Kong, and K.-S. Lee, Appl. Phys. Lett. 87, 154108 (2005).

${ }^{11}$ S. H. Park, T. W. Lim, D. Y. Yang, J. H. Jeong, K. D. Kim, K.-S. Lee, and H. J. Kong, Appl. Phys. Lett. 88, 203105 (2006).

${ }^{12}$ S. H. Park, T. W. Lim, D. Y. Yang, N. C. Cho, and K.-S. Lee, Appl. Phys. Lett. 89, 173133 (2006).

${ }^{13}$ D. Y. Yang, S. H. Park, T. W. Lim, H. J. Kong, S. W. Yi, H. K. Yang, and K.-S. Lee, Appl. Phys. Lett. 90, 013113 (2007).

${ }^{14}$ A. W. Adamson and A. P. Gast, Physical Chemistry of Surfaces, Fifth ed. (Wiley, New York, 1997), Chap. 1, pp. 7-10.

${ }^{15}$ D.-G. Choi, J. H. Jeong, Y. S. Sim, E. S. Lee, W. S. Kim, and B. S. Bae, Langmuir 21, 9390 (2005). 